\title{
Hybridized wavefront shaping for high-speed, high-efficiency focusing through dynamic diffusive media
}

\author{
Ashton S. Hemphill \\ Jian Wei Tay \\ Lihong V. Wang
}




\title{
Hybridized wavefront shaping for high-speed, high-efficiency focusing through dynamic diffusive media
}

\author{
Ashton S. Hemphill, ${ }^{a}$ Jian Wei Tay, ${ }^{a, \dagger}$ and Lihong V. Wang ${ }^{\mathrm{a}, *}$ \\ aWashington University in St. Louis, Optical Imaging Laboratory, Department of Biomedical Engineering, One Brookings Drive, Saint Louis, \\ Missouri 63130, United States
}

\begin{abstract}
One of the prime limiting factors of optical imaging in biological applications is the diffusion of light by tissue, which prevents focusing at depths greater than the optical diffusion limit (typically $\sim 1 \mathrm{~mm}$ ). To overcome this challenge, wavefront shaping techniques that use a spatial light modulator (SLM) to correct the phase of the incident wavefront have recently been developed. These techniques are able to focus light through scattering media beyond the optical diffusion limit. However, the low speeds of typically used liquid crystal SLMs limit the focusing speed. Here, we present a method using a digital micromirror device (DMD) and an electro-optic modulator $(E O M)$ to measure the scattering-induced aberrations, and using a liquid crystal SLM to apply the correction to the illuminating wavefront. By combining phase modulation from an EOM with the DMD's ability to provide selective illumination, we exploit the DMD's higher refresh rate for phase measurement. We achieved focusing through scattering media in less than $8 \mathrm{~ms}$, which is sufficiently short for certain in vivo applications, as it is comparable to the speckle correlation time of living tissue. ๑ 2016 Society of Photo-Optical Instrumentation Engineers (SPIE) [DOI: 10.1117/1.JBO.21.12.121502]
\end{abstract}

Keywords: high-speed wavefront shaping; wavefront shaping; active or adaptive optics; dynamic media; turbid media. Paper 160172SSPR received Mar. 18, 2016; accepted for publication May 17, 2016; published online Sep. 14, 2016.

\section{Introduction}

Light plays a critical role in high-resolution, high-sensitivity imaging, ${ }^{1-3}$ as well as in applications, which require the precise delivery of energy, such as optogenetics, ${ }^{4}$ microsurgery, ${ }^{5}$ and photothermal therapy. ${ }^{6}$ However, light experiences severe scattering in biological tissue, which randomizes the phase of the optical wavefront. The self-interference of the scrambled wavefront results in a speckle field, as shown in Fig. 1(a). Consequently, optical scattering prevents focusing using lenses at depths beyond the optical diffusion limit $(\sim 1 \mathrm{~mm}$ in soft tissue $^{7}$ ), resulting in greatly limited feasible depths of both optical imaging and therapeutic techniques. To overcome this limitation, optical phase conjugation (OPC) and wavefront shaping (WFS) have recently been developed to focus light beyond the optical diffusion limit. Both of these methods function by modifying the wavefront of the incident illumination to correct for the aberrations caused by scattering, as illustrated in Fig. 1(b).

The approaches used to obtain the optimized wavefront differ between these two methods. In OPC, the wavefront correction is determined directly by measuring the wavefront of the scattered light outside the diffusive medium. To focus within the medium, a "guide star" is utilized to selectively tag the light travelling through the desired target location. These guide stars can be real (e.g., fluorescent particles ${ }^{8-10}$ ) or virtual (e.g., using focused ultrasound to frequency-shift the light $\left.{ }^{11-13}\right)$. The measured diffused wavefront is then phase-conjugated (i.e., its phase is shifted by $\pi$ ) to form a focus at the guide star location upon propagation back through the scattering medium. Both digital

*Address all correspondence to: Lihong V. Wang, E-mail: Ihwang@ wustl.edu ${ }^{\dagger}$ Present address: University of Colorado, Department of Electrical, Computer and Energy Engineering, Boulder, Colorado 80309, United States and analog OPC have been demonstrated. In digital OPC, a CCD camera records the scattered wavefront, while a spatial light modulator (SLM) produces the phase-conjugated wavefront. High enhancement factors (defined as the ratio of the focused light intensity over the initial light intensity) have been demonstrated using digital OPC. . $^{8,13}$

However, digital OPC is slow. In general, about $100 \mathrm{~ms}$ is required to record and reproduce the optimized wavefront ${ }^{14}$ due to long camera acquisition and data transfer, as well as the low refresh rate (typically $<300 \mathrm{~Hz}$ ) of the liquid crystal on silicon (LCoS) SLMs used. This long optimization time is especially problematic for use with living tissue, which is dynamic, meaning inhomogeneities shift and cause scattering to rapidly decorrelate, on the order of milliseconds. ${ }^{15,16}$ Thus, for the collected phase information to be real-time accurate, wavefront optimization should ideally be completed within $10 \mathrm{~ms},{ }^{16}$ much too fast for the low refresh rate of LCoS SLMs.

Alternatively, analog OPC uses a photorefractive crystal to record and produce the phase-conjugated wavefront holographically. ${ }^{11,17-19}$ This method is much faster; typically, only $10 \mathrm{~ms}$ is needed to generate the phase-conjugated wavefront. ${ }^{19}$ However, as the photorefractive hologram is erased on readout, the amount of light that can be focused through the medium is less than the amount of light used during the recording process (i.e., the enhancement factor is less than unity), reducing its utility in many applications despite the recent demonstration of power gain. ${ }^{20}$

In contrast to OPC, WFS uses multiple measurements to identify the corrected wavefront. ${ }^{21-28}$ Here, the SLM is used to modify the wavefront of the illuminating beam. To obtain the corrected 


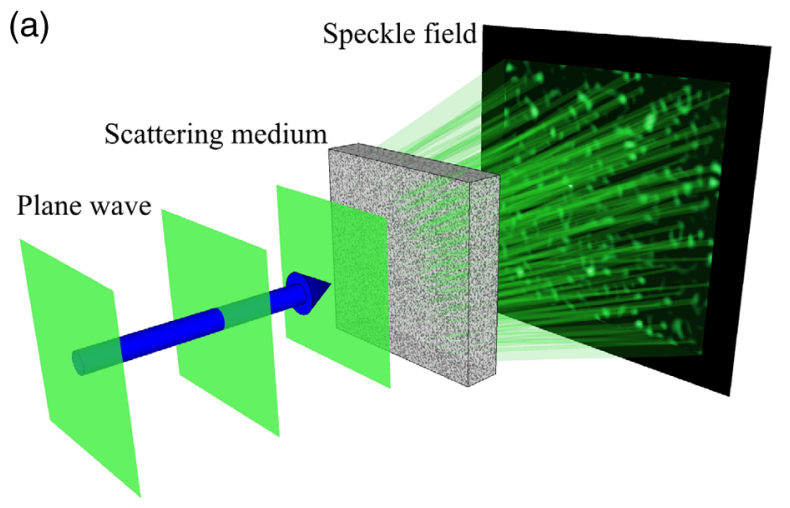

(b)

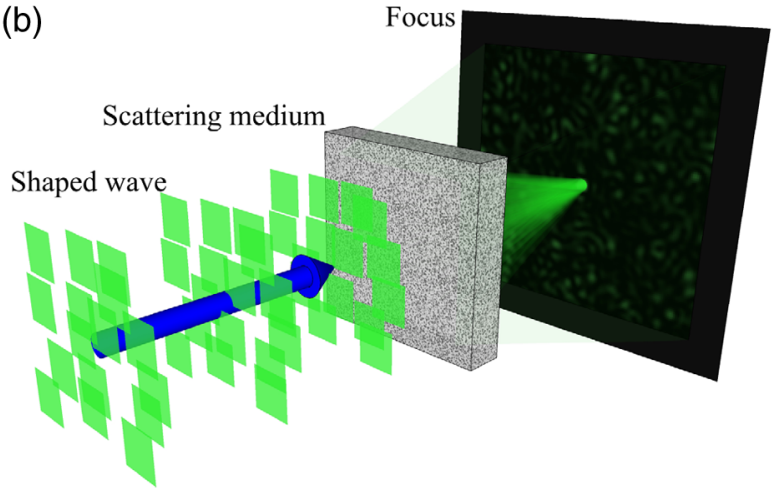

Fig. 1 Illustration of focusing through scattering media by wavefront shaping. (a) The phase scrambling of a plane wave by travelling through a scattering medium results in a speckle field. (b) In wavefront shaping, the wavefront is corrected for scattering and forms a focus at the target location.

wavefront, an iterative or basis optimization algorithm can be used. $^{21-28}$ The optical intensity at the target location is measured and used as feedback to the optimization algorithm. The algorithm then searches for the optimal SLM pattern to maximize the feedback signal, thereby forming a focus through the diffusive medium. However, current WFS techniques that also suffer from low optimization speeds as LCoS SLMs, which must be updated for each phase map, are typically utilized.

To increase optimization speeds, digital micromirror devices (DMDs), which can have update rates of several tens of kilohertz, may be used. ${ }^{21,26,27}$ However, these devices provide binary amplitude-modulation, which has been shown to be five times less efficient than phase correction. ${ }^{27,29}$ DMDs can be used to provide phase modulation, for example, by utilizing off-axis holography. ${ }^{22}$ However, since only the first diffraction order contains phase information, $\sim 80 \%$ of the total illumination to the DMD is sacrificed, reducing overall illumination during both measurement and focusing. ${ }^{22,27,29}$ As biological applications already suffer from low signal-to-noise ratios (SNRs), this limitation makes use of a DMD for wavefront correction, unattractive in high-sensitivity applications.

To overcome these issues, we demonstrate a hybrid WFS method, which utilizes both an SLM and a DMD. In contrast to previous methods, we do not use off-axis holography and thus maintain full illumination. Instead, the DMD is used to selectively illuminate one superpixel of the SLM at a time. To determine the optimal phase of each superpixel, an electro-optic modulator (EOM) is used to phase-shift the beam through $2 \pi$. The optimized phase pattern is then displayed on the SLM. In this hybrid approach, use of the DMD for measurement greatly reduced the optimization time while the SLM provided high-efficiency phase-based wavefront correction. We were able to optimize the optical wavefront with 64 modes in less than $8 \mathrm{~ms}$, which is within the speckle correlation time in living tissue.

\section{Methods}

The experimental layout of our hybrid system is shown in Fig. 2. Illumination was provided by a $5-\mathrm{W}$ continuous wave laser (Verdi V-5, Coherent, United States) at $532 \mathrm{~nm}$. The beam was vertically polarized before being split into the sample and reference arms using the first nonpolarizing beamsplitter. Along the sample arm, the beam was expanded by the first pair of lenses to fully illuminate the DMD (DLP 7000, Texas

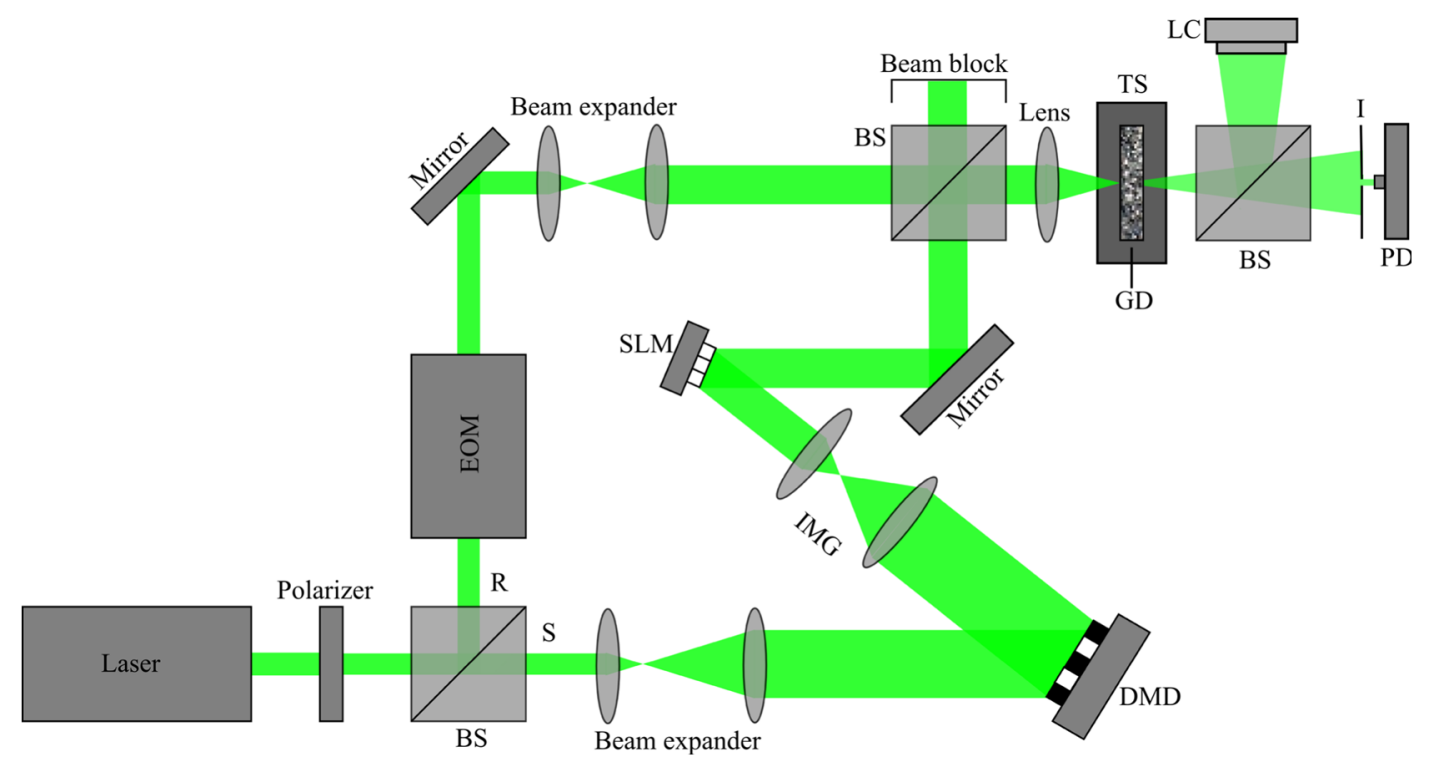

Fig. 2 Schematic of the optical system. BS, beam splitter; DMD, digital micromirror; EOM, electro-optic modulator; GD, ground glass diffuser; I, iris; IMG, 4f imaging system; LC, line camera; PD, photodiode; R, reference arm; S, sample arm; SLM, spatial light modulator; TS, translation stage. 
Instruments, United States). A second pair of lenses (LA1433A, Thorlabs, United States and KPX091AR.14, Newport, United States) was used to image the DMD to the surface of the SLM (HSP256-0532, Meadowlark Optics, United States), with each superpixel of the DMD corresponding to an SLM superpixel. These lenses were selected to adjust the beam size while providing sufficient focal length for angular matching of the SLM and DMD. For the following experiments, we divided both the DMD and the SLM into 64 superpixels.

Along the reference arm, the beam was directed through a phase modulating EOM (350-105, Conoptics, United States). The EOM was driven with a $1.25 \mathrm{Vpp}$ ramp function (corresponding to a $2 \pi$ phase shift), supplied at $62.5 \mathrm{kHz}$, three times per iteration by a function generator (DS345, Stanford Research Systems, United States) and amplified 375× using

(a)

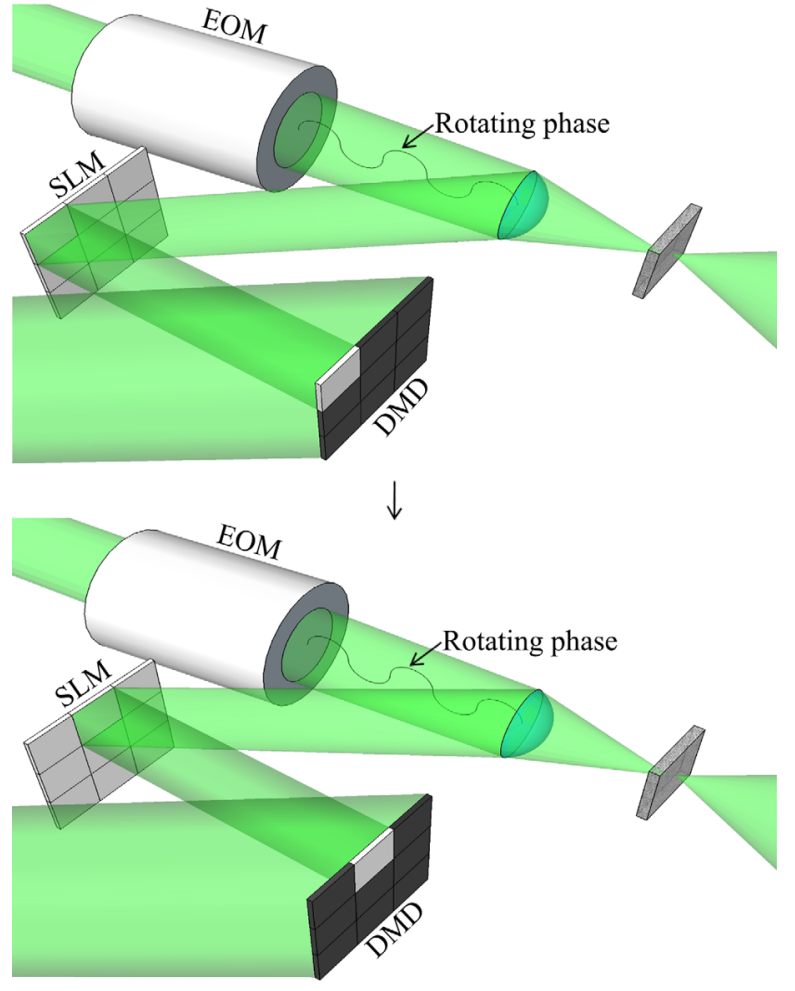

(b)

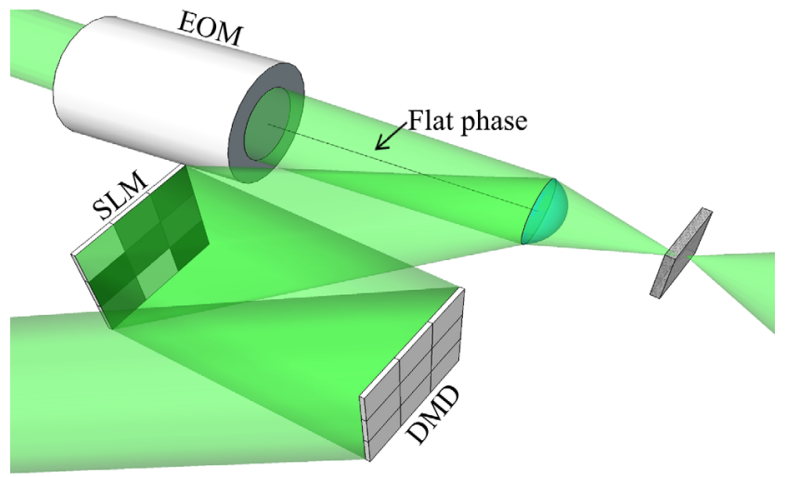

Fig. 3 Illustration of the hybrid system in operation. (a) During the measurement stage, the DMD illuminates the SLM one superpixel at a time, while the EOM modulates the phase. A flat phase pattern is displayed on the SLM. (b) During the readout step, the DMD pixels are turned on, and the optimized phase pattern is displayed on the SLM. a high-voltage linear amplifier (M302RM, Conoptics, United States). The beam was then expanded by a pair of lenses to match the size of the sample beam. The beams in the two arms were then recombined using a second nonpolarizing beamsplitter. The combined beams were then focused by a lens (LA1951-A, Thorlabs) onto the ground glass diffuser (DG10220, Thorlabs), serving as the scattering medium. The beam was allowed to propagate $\sim 25 \mathrm{~cm}$ beyond the diffuser, before a single speckle grain was isolated using an adjustable iris (SM1D12, Thorlabs), which was mounted on the chassis of a photodiode (PDA31A, Thorlabs). The signal from the photodiode was amplified by $40 \mathrm{~dB}$, then digitized using a data acquisition (DAQ) card (ATS9462, AlazarTech, Canada) at $10 \mathrm{MS} / \mathrm{s}$ to provide 160 phase levels for direct optimization. To verify the formation of the optical focus, a beamsplitter was used to divert a portion of the illumination from the scattering media to a CCD camera (Grasshopper 3, Point Grey, Canada), which was placed the same distance from the beamsplitter as the photodiode. The EOM driver, DAQ card, and DMD were triggered at $18.85 \mathrm{kHz}$ by a second DAQ card (NI 6321, National Instruments) and a function generator (DG4162, Rigol Technologies, China).

A single focusing cycle consisted of three steps: measurement, calculation, and display. As shown in Fig. 3, during the measurement stage, the DMD sequentially provided illumination to each superpixel. The signal from the photodiode was recorded as the EOM modulated the phase of the reference beam through a $2 \pi$ phase shift. Throughout this process, a flat pattern was displayed on the SLM. After the intensity profiles were measured for each superpixel, the optimal phase of each superpixel was determined by identifying the phase corresponding to the maximum signal. During readout, the resulting phase pattern was then displayed on the SLM while the DMD was turned fully on to provide complete illumination of the SLM. In this way, our method performs phase measurements at DMD speeds, with only a single refresh of the SLM required.

\section{Results}

There are two main requirements for the WFS system to be useful for applications with living tissue: (1) the wavefront should be optimized within the in vivo speckle correlation time $\left(<10 \mathrm{~ms}^{16}\right)$ and (2) the enhancement factor should be greater than unity. To demonstrate that our system meets these

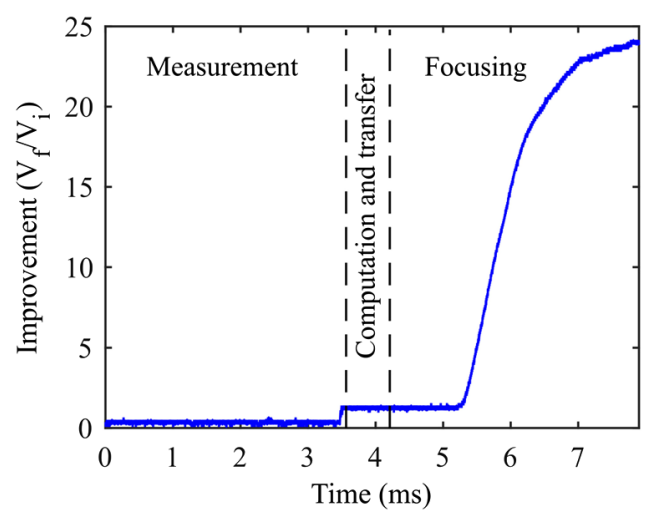

Fig. 4 Illustration of the timing of the system. Measurement of the transmission matrix requires $3.56 \mathrm{~ms}$. Transfer of the data to the computer and computation of the phase map requires $0.65 \mathrm{~ms}$. Transfer and display of the optimized phase map required $3.69 \mathrm{~ms}$ due to the SLM update rate. 


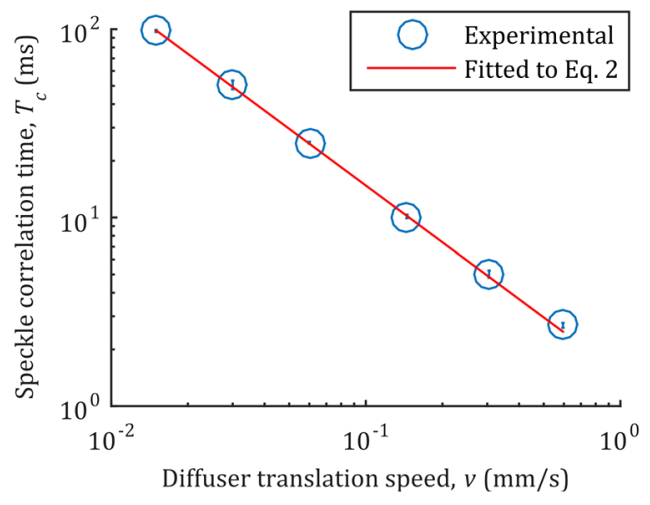

Fig. 5 Relationship between speckle correlation time and translation speed of the diffuser. The error bars illustrate the standard error over 100 measurements, each at different locations on the ground glass diffuser.

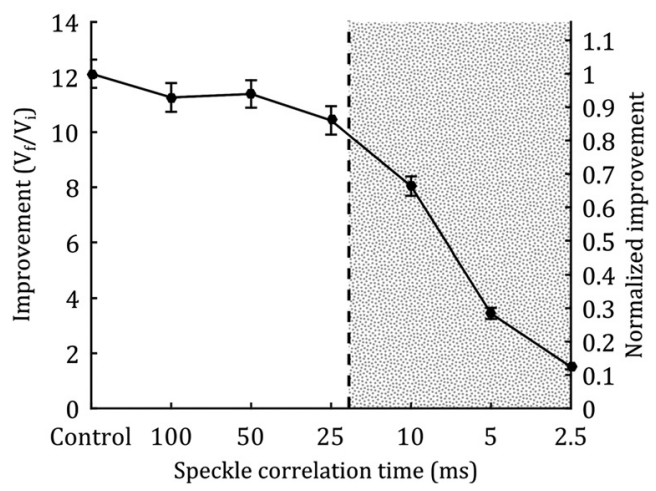

Fig. 6 Focusing through a moving diffuser. The control point represents a stationary diffuser with $T_{\mathrm{c}}>10,000 \mathrm{~ms}$. At speckle correlation times of 10 and $5 \mathrm{~ms}$, enhancement is $66.3 \%$ and $28.4 \%$ of the control, respectively. Improvement from control to $T_{\mathrm{c}}$ of $25 \mathrm{~ms}$ (unshaded region) does not statistically differ from one another. Improvement in the shaded region ( $T_{\mathrm{c}}$ of 10,5 , and $2.5 \mathrm{~ms}$ ) decreases and is significantly different $(p<0.02)$ from all other groups. The error bars show the standard error over 100 measurements.

(a)

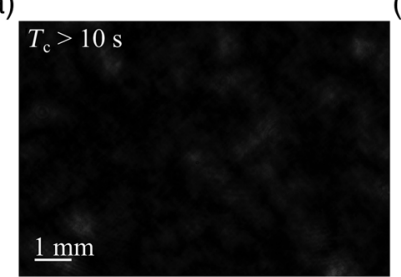

(e)

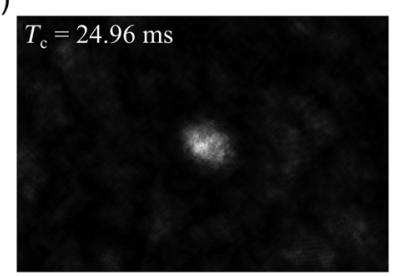

(b)

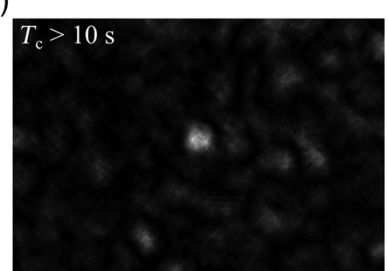

(f)

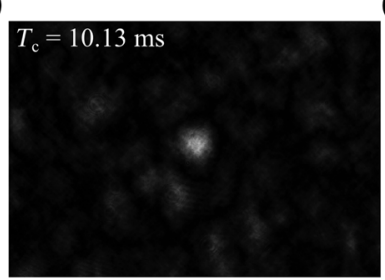

conditions, we ran the optimization routine first with the ground glass diffuser held stationary. For 64 superpixels, we measured a runtime, averaged over 10 focusing cycles, of $7.89 \pm 0.04 \mathrm{~ms}$, which is below $10 \mathrm{~ms}$. The timing of optimization over the course of a focusing cycle is demonstrated in Fig. 4. To our knowledge, this is the fastest demonstration of phase-based WFS to date.

The averaged enhancement factor was $19.1 \pm 1.4$, which is $\sim 37.8 \%$ of the theoretical maximum enhancement $\left(\eta_{\max }\right)$, which is given below ${ }^{21}$ :

$\eta_{\max }=\frac{\pi}{4}\left(\frac{N}{M}-1\right)+1$,

where $N$ is the number of superpixels and $M$ is the number of speckle grains at the detector. The achieved enhancement factor is in agreement with the values obtained by previous studies ${ }^{21,23}$ with regard to the enhancement as a percentage of the theoretical maximum.

\subsection{Tissue-Mimicking Phantom Experiment}

We also demonstrate the capability of our hybrid system to focus through scattering media with varied speckle correlation times, particularly those similar to living tissue. To vary the speckle correlation time, the ground glass diffuser was mounted on a variable velocity motorized translation stage (CONEX-LTAHS, Newport), as shown in Fig. 2. A line camera (Aviiva M2, Atmel, United States) was then used to record the speckle grain field as the stage was translated at different speeds. The temporal correlation coefficient between the frames was calculated, with the first frame used as the reference. The corresponding speckle correlation times, defined as the time for the correlation coefficient to reach $1 / \mathrm{e}^{2}$, were then obtained by fitting the correlation coefficient of each frame to a Gaussian function. ${ }^{19}$ The measured speckle correlation times and the standard error of the measurements are shown in Fig. 5.

The measured speckle correlation times were fitted to

$T_{c}=\frac{d_{b}}{v}$,

(c)

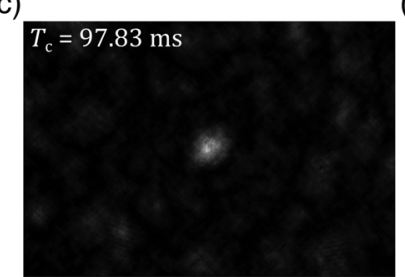

(d)

(g)

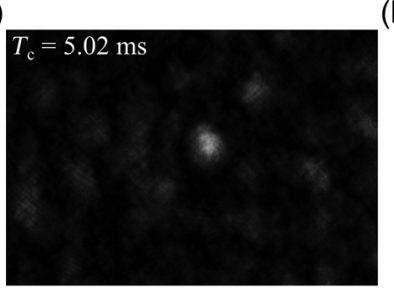

(h)

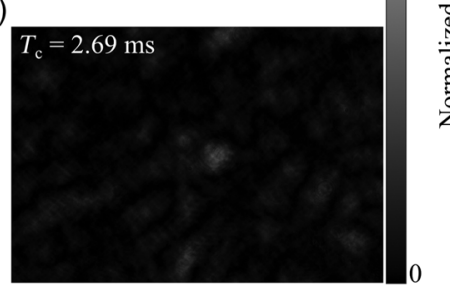

Fig. 7 Representative images of the foci formed when ground glass diffuser was (a) held stationary $\left(T_{\mathrm{c}}>10 \mathrm{~s}\right)$ with no wavefront optimization, (b) held stationary $\left(T_{\mathrm{c}}>10 \mathrm{~s}\right)$ with phase optimization, (c) translated at $0.015 \mathrm{~mm} / \mathrm{s}\left(T_{\mathrm{c}}=97.83 \mathrm{~ms}\right)$ with phase optimization, (d) translated at $0.03 \mathrm{~ms}$ $\left(T_{\mathrm{c}}=50.66 \mathrm{~ms}\right), \quad$ (e) translated at $0.060 \mathrm{~mm} / \mathrm{s}\left(T_{\mathrm{c}}=24.96 \mathrm{~ms}\right),(\mathrm{f})$ translated at $0.145 \mathrm{~mm} / \mathrm{s}$ $\left(T_{\mathrm{c}}=10.13 \mathrm{~ms}\right)$, $(\mathrm{g})$ translated at $0.305 \mathrm{~mm} / \mathrm{s}\left(T_{\mathrm{c}}=5.02 \mathrm{~ms}\right)$, and $(\mathrm{h})$ translated at $0.595 \mathrm{~mm} / \mathrm{s}$ $\left(T_{\mathrm{c}}=2.69 \mathrm{~ms}\right)$. The images were normalized to the local maximum intensity. 
where $T_{\mathrm{c}}$ is the speckle correlation time in milliseconds, $d_{b}=$ $1.47 \mu \mathrm{m}$ is the estimated size of the speckle grain at the surface of the scattering medium, ${ }^{19}$ and $v$ is the translation speed of the medium.

We then used the hybrid system to focus light through the moving diffuser at speckle correlation times of 100, 50, 25, 10,5 , and $2.5 \mathrm{~ms}$. A stationary control was also used, having a measured correlation time of over $10,000 \mathrm{~ms}$. We repeated the measurement 100 times for each speckle correlation time, at separate locations on the diffuser to produce different speckle grain fields.

For the control, we measured an average enhancement of $12.1 \pm 0.5$ (Fig. 6). As shown in Fig. 6, when the correlation time was much larger than the runtime, there was no significant difference in improvement. As correlation time approached and dropped below the runtime, the improvement was decreased proportionally, but remained greater than unity at all times. Furthermore, at correlation times of 10 and $5 \mathrm{~ms}$, which are within the speckle correlation time of living tissue, the enhancement was $66.3 \%$ and $28.4 \%$ of the control.

To verify, the resulting foci were imaged with the CCD camera and are shown in Fig. 7. In each image, a clearly distinguishable focus was observed. The intensity of the foci was also lowered as the correlation time decreased, in agreement with the results presented in Fig. 6.

\section{Discussion and Conclusions}

There currently exist a variety of methods for focusing through scattering media. However, current techniques are hindered by the rapid speckle decorrelation of tissue in vivo. This has caused a necessary compromise between speed and utility in the majority of approaches, with detrimental effects, such as loss of fluence or gain seen as unfortunate requisites for high-speed focusing. LCoS SLMs have insufficient update rates to function effectively in WFS or digital OPC. Analog methods allow highspeed optimization, but result in gains of less than unity. A DMD, with its higher refresh rate, is ideal, but provides about five times less enhancement for an equivalent number of modes compared to the phase-based WFS of SLMs. While using a DMD to obtain phase modulation results in higher speeds, this holographic technique is inefficient, causing a loss of $\sim 80 \%$ of the incident illumination to the DMD during both measurement and final optimized focusing.

In this report, we have demonstrated a novel hybrid technique, which allows us to take advantage of the high-speed DMD while also using an LCoS SLM for high efficiency. By using the EOM to modulate the phase, we were able to take advantage of the high refresh rate of the DMD, as the optimal phase for each superpixel was obtained with just a single update to the DMD. By contrast, previous implementations of highspeed, phase-based WFS required the pattern to be updated at least three or four times per superpixel. ${ }^{21}$

Using this method, we have demonstrated the speed and efficacy of the hybrid system by focusing light though stationary scattering media and achieved high enhancement factors of up to $38 \%$ of the maximum theoretical improvement with a runtime of under $8 \mathrm{~ms}$, within the range of in vivo speckle correlation times. To better simulate the motion of living tissue, the ground glass diffuser was then mounted on a motorized stage, to demonstrate focusing at speckle correlation times similar to in vivo tissue. We showed that enhancement was greater than unity at all tested correlation times and confirmed these results by using a CCD to directly image the formed foci.

The speed of the system is dependent on the number of superpixels used, as well as the time needed to update the SLM. We found that the optimization required $\sim 55 \mu$ s per superpixel (constrained by the speed of the DMD), as well as about $4 \mathrm{~ms}$ to update the SLM display. Because of this, 64 superpixels were used to obtain a runtime of $<10 \mathrm{~ms}$. This runtime should be sufficient to allow our method to focus light, for example, through several millimeters of brain tissue. ${ }^{30}$

In this experiment, we used an aperture at the photodiode to detect only one speckle grain. Our hybrid system should also be compatible with the use of internal guide stars, such as photoacoustic or ultrasonic feedback for focusing within turbid media. ${ }^{11,13,23,25}$ However, when focusing in living tissue, multiple speckle grains could fall within the area of detection due to the optical diffraction limited speckle size $(\lambda / 2)$. In this case, as shown in Eq. (1), the maximum theoretical enhancement factor would be reduced. To recover the enhancement, more superpixels could be used, at the cost of increased runtime.

Another important consideration is that as the number of superpixels is increased, the amount of light per pixel is reduced. Hence, the SNR of the feedback signal may become an issue with large numbers of superpixels. In this case, it may become beneficial to use illumination patterns generated using the Hadamard basis, which provides greater illumination compared to selectively illuminating the SLM one superpixel at a time. $^{23,29,31}$ Both the patterns utilized and number of input modes may be changed in the current system without modifications to the optical layout. However, in our proof-of-principle study, we did not suffer from low SNR; therefore, use of the Hadamard basis was unnecessary.

In summary, our hybrid method offers significant advantages over previous WFS techniques. By utilizing a DMD in tandem with the SLM, the hybrid system functions at the speed of the DMD while maintaining on-axis phase modulation. This high speed allows for extremely fast optimization, demonstrated to be capable of focusing through diffusive media with correlation times within range of living tissue. Combined with the high efficiency of the phase-based on-axis layout, the hybrid system shows potential to advance biomedical optics by increasing the focusing depth through and, possibly, in biological tissue. While factors such as degrees of freedom, DMD damage threshold, and further speed enhancement require refinement, once the current technical challenges are solved, our hybrid technique has anticipated applications in optogenetics, photothermal therapy, microsurgery, and more.

\section{Acknowledgments}

We thank Jinyang Liang for discussions regarding SLM and DMD alignment, Yan Liu for discussion regarding the dynamic diffusive media experiment, and Seema Dahlheimer for editing the manuscript. This work was supported in part by the National Institute of Health grants DP1 EB016986 (NIH Director's Pioneer Award) and R01 CA186567 (NIH Director's Transformative Research Award). L.V.W. has a financial interest in Microphotoacoustics, Inc. and Endra, Inc., which, however, did not support this work.

\section{References}

1. W. Denk, J. Strickler, and W. Webb, "Two-photon laser scanning fluorescence microscopy," Science 248(4951), 73-76 (1990). 
2. J. Pawley, Handbook of Biological Confocal Microscopy, Springer, New York City (2006).

3. D. Huang et al., "Optical coherence tomography," Science 254(5035), 1178-1181 (1991).

4. L. Fenno, O. Yizhar, and K. Deisseroth, "The development and application of optogenetics," Ann. Rev. Neurosci. 34(1), 389-412 (2011).

5. M. F. Yanik et al., "Neurosurgery: functional regeneration after laser axotomy," Nature 432(7019), 822-822 (2004).

6. E. I. Galanzha et al., "In vivo fiber-based multicolor photoacoustic detection and photothermal purging of metastasis in sentinel lymph nodes targeted by nanoparticles," J. Biophotonics 2(8-9), 528-539 (2009).

7. L. V. Wang and H. I. Wu, Biomedical Optics: Principles and Imaging, John Wiley \& Sons, Inc., New York City (2009).

8. C. L. Hsieh et al., "Digital phase conjugation of second harmonic radiation emitted by nanoparticles in turbid media," Opt. Express 18(12), 12283-12290 (2010).

9. I. M. Vellekoop and C. M. Aegerter, "Scattered light fluorescence microscopy: imaging through turbid layers," Opt. Lett. 35(8), 12451247 (2010).

10. I. M. Vellekoop, M. Cui, and C. Yang, "Digital optical phase conjugation of fluorescence in turbid tissue," Appl. Phys. Lett. 101(8), 081108 (2012).

11. X. Xu, H. Liu, and L. V. Wang, "Time-reversed ultrasonically encoded optical focusing into scattering media," Nat. Photonics 5(3), 154-157 (2011).

12. K. Si, R. Fiolka, and M. Cui, "Fluorescence imaging beyond the ballistic regime by ultrasound-pulse-guided digital phase conjugation," Nat. Photonics 6(10), 657-661 (2012).

13. Y. M. Wang et al., "Deep-tissue focal fluorescence imaging with digitally time-reversed ultrasound-encoded light," Nat. Coтmun. 3, 928 (2012).

14. E. H. Zhou et al., "Focusing on moving targets through scattering samples," Optica 1(4), 227-232 (2014).

15. A. Lev and B. Sfez, "In vivo demonstration of the ultrasound-modulated light technique," J. Opt. Soc. Am. A 20(12), 2347-2354 (2003).

16. M. Jang et al., "Relation between speckle decorrelation and optical phase conjugation (OPC)-based turbidity suppression through dynamic scattering media: a study on in vivo mouse skin," Biomed. Opt. Express 6(1), 72-85 (2015).

17. Z. Yaqoob et al., "Optical phase conjugation for turbidity suppression in biological samples," Nat. Photonics 2(2), 110-115 (2008).
18. M. Cui, E. J. McDowell, and C. Yang, "An in vivo study of turbidity suppression by optical phase conjugation (TSOPC) on rabbit ear," Opt. Express 18(1) 25-30 (2010).

19. Y. Liu et al., "Optical focusing deep inside dynamic scattering media with near-infrared time-reversed ultrasonically encoded (TRUE) light," Nat. Commun. 6, 5904 (2015).

20. C. Ma et al., "Single-exposure optical focusing inside scattering media using binarized time-reversed adapted perturbation," Optica 2(10), 869-876 (2015).

21. I. M. Vellekoop and A. P. Mosk, "Focusing coherent light through opaque strongly scattering media," Opt. Lett. 32(16), 2309-2311 (2007).

22. D. B. Conkey, A. M. Caravaca-Aguirre, and R. Piestun, "High-speed scattering medium characterization with application to focusing light through turbid media," Opt. Express 20(2), 1733-1740 (2012).

23. T. Chaigne et al., "Controlling light in scattering media non-invasively using the photoacoustic transmission matrix," Nat. Photonics 8(1), 58-64 (2014).

24. J. W. Tay et al., "Ultrasonically encoded wavefront shaping for focusing into random media," Sci. Rep. 4, 3918 (2014).

25. P. Lai et al., "Photoacoustically guided wavefront shaping for enhanced optical focusing in scattering media," Nat. Photonics 9(2), 126-132 (2015).

26. A. M. Caravaca-Aguirre et al., "Real-time resilient focusing through a bending multimode fiber," Opt. Express 21(10), 12881-12887 (2013).

27. D. Akbulut et al., "Focusing light through random photonic media by binary amplitude modulation," Opt. Express 19(5), 4017-4029 (2011).

28. A. P. Mosk et al., "Controlling waves in space and time for imaging and focusing in complex media," Nat. Photonics 6(5), 283-292 (2012).

29. J. W. Tay, J. Liang, and L. V. Wang, "Amplitude-masked photoacoustic wavefront shaping and application in flowmetry," Opt. Lett. 39(19), 5499-5502 (2014).

30. J. Brake, M. Jang, and C. Yang, "Analyzing the relationship between decorrelation time and tissue thickness in acute rat brain slices using multispeckle diffusing wave spectroscopy," J. Opt. Soc. Am. A 33(2), 270-275 (2016).

31. I. M. Vellekoop and A. P. Mosk, "Phase control algorithms for focusing light through turbid media," Opt. Commun. 281(11), 3071-3080 (2008).

Biographies for the authors are not available. 